\title{
Schlechte Evidenz für Pharmakotherapie
}

Fragestellung: Welche pharmakologischen Behandlungsoptionen psychiatrischer Symptome gibt es bei der frontotemporalen Demenz (FTD)?

Hintergrund: Patienten mit FTD weisen aufgrund der spezifischen Ätiologie der Erkrankung mit Degeneration des Frontalund Temporallappens bestimmte psychiatrische Symptome häufiger auf als Patienten mit anderen Demenzformen. Der neurodegenerative Prozess bei der FTD ist multifaktorieller als bei der Alzheimer-Demenz, letztlich kommt es jedoch zur neurotoxischen Ablagerung von Tauproteinen, TDP-43 (Transactivation response element DNA-binding Protein $43 \mathrm{kDa}$ ) oder anderen Kopathogenen. Eine familiäre Häufung der FTD wird in 40 - 50\% der Fälle beobachtet. Meist handelt es sich um Mutationen im mikrotubulusassoziierten Protein Tau, Progranulin-Gen oder um Expansionen von Hexanukleotiden auf Chromosom 9 im open reading frame 72 (C9ORF72). Klinisch sind Patienten mit FTD unter anderem häufiger enthemmt und psychomotorisch unruhig als Patienten mit Alzheimer-Demenz. Auch sind Schlaf- und Essstörungen häufiger. Diese klinischen Beobachtungen haben zu der Vermutung geführt, dass die psychiatrischen Symptome bei FTD durch andere Neurotransmitterstörungen als bei der Alzheimer-Demenz verursacht sind, bei der vorwiegend eine Störung der cholinergen Transmission vorliegt. Bei der FTD beste-

Buoli M, Serati M, Caldiroli A et al. Pharmacological management of psychiatric symptoms in frontotemporal dementia: A systematic review. J Geriatr Psychiatry Neurol 2017; 30: $162-9$ hen dagegen Störungen im Serotonin- und Dopaminsystem, während das Acetylcholinsystem relativ intakt ist. PET-Studien und postmortale Untersuchungen zeigten einen weitgehenden
Verlust der Serotoninrezeptoren. Eine direkte Korrelation zwischen der dopaminergen Neurotransmission und Verhaltensstörungen wurde berichtet. Zusammenfassend legen diese Daten nahe, dass die psychiatrischen Symptome bei der FTD eine spezifische Ätiologie haben und schwerwiegender als bei anderen Demenzformen sein können. Pflegende Angehörige sind damit einer höheren Belastung ausgesetzt als Angehörige bei anderen Demenzformen. Daher sollte stärker nach effektiven Behandlungsmöglichkeiten der Verhaltensstörungen bei FTD gesucht werden.

Patienten und Methodik: Es handelt sich um eine systematische Übersichtsarbeit von 29 englischsprachigen Publikationen, die in den gängigen Datenbanken identifiziert wurden.

Ergebnisse: Von den Antidementiva war Donepezil unwirksam oder verstärkte die psychischen Symptome bei der FTD sogar, während eine offene Studie mit Rivastigmin eine Besserung zeigte. Galantamin und Memantin erzielten keinen Effekt. Im Hinblick auf den Einsatz von Antidepressiva belegten kleinere Studien einen positiven, teils dosisabhängigen Effekt für Trazodon. Mehrere kleine Studien mit Serotonin-Wiederaufnahmehemmern (SSRI) zeigten teils positive Effekte.

Schlussfolgerungen: Im Gegensatz zu Häufigkeit und Schwere der psychischen Probleme bei der FTD liegt eine sehr schlechte Evidenz zur pharmakologischen Behandlung vor. Klassische Antidementiva inklusive Memantin sind unwirksam. Im Gegensatz dazu waren die Studien mit SSRI mit Ausnahme von Paroxetin positiv, was die Autoren mit dem anticholinergen Effekt von Paroxetin erklären. Es handelte sich aber durchweg um kleine offene Studien mit nur zehn bis 20 Patienten.

\section{- Kommentar von Markus Weih, Nürnberg}

\section{Für den Einsatz von SSRI gäbe es sogar eine vernünftige Erklärung}

Während es bei der Demenz vom Alzheimer-Typ mit den Cholinesterasehemmern und Memantin ein kleines, älteres Arsenal von mäßig wirksamen Antidementiva gibt, sieht es für Patienten mit der FTD weiter düster aus: Es gibt weder für einen kausalen Ansatz noch für eine symptomatische Behandlung der so belastenden psychischen Symptome eine effektive Therapie, alle Studien sind erschreckend klein.

Die Arbeitsgruppe um M. Buoli aus Mailand hat sich nun noch einmal die Mühe gemacht, die aktuelle Studienlage zusammenzutragen. Sie kommen dabei im Wesentlichen zu demselben Schluss wie die aktuellen deutschen S3-Leitlinien zur Demenz: Es gibt weiterhin keine überzeugende Evidenz für die symptomatische pharmakologische Behandlung bei der FTD. Antipsychotika müssen sehr vorsichtig eingesetzt werden. Man sollte aber auf Depression, Apathie und Impulsivität achten. Hier kann sich im Einzelfall ein Therapieversuch mit einem SSRI wie Ser- tralin oder Escitalopram lohnen. Berücksichtigt man die wenigen Arbeiten, die eine gestörte serotonerge Neurotransmission nahelegten, gibt es für diesen Ansatz sogar eine vernünftige Erklärung, jenseits von großen randomisierten Studien.

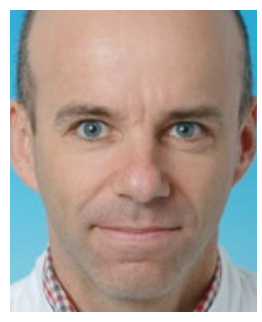

Prof. Dr. med. Markus Weih, Nürnberg

Facharzt für Neurologie, Psychiatrie und Psychotherapie, Nervenärztliche Gemeinschaftspraxis E-Mail:weih@nervenaerzteallersbergerstrasse.de 